\title{
Numerical and Experimental Investigation of the Sealing Effect of a Specific Labyrinth Seal Structure
}

\author{
Shiping Yang (D), Bosi Tan (1), and Xiaofen Deng \\ School of Mechanical Engineering, Xiangtan University, Xiangtan 411100, China \\ Correspondence should be addressed to Shiping Yang; yspxtu@yeah.net
}

Received 7 April 2019; Revised 20 May 2019; Accepted 28 May 2019; Published 24 June 2019

Guest Editor: Xizhong An

Copyright (C) 2019 Shiping Yang et al. This is an open access article distributed under the Creative Commons Attribution License, which permits unrestricted use, distribution, and reproduction in any medium, provided the original work is properly cited.

\begin{abstract}
A numerical simulation and experimental study were performed to investigate the sealing effect of the labyrinth seal used in a wheelside reducer. A three-dimensional computational fluid dynamics model was established based on the labyrinth structure. The effects of the temperature and viscosity of lubricant, rotor speed, pressure ratio, clearance, and cavity size of the sealing structure on the sealing were analyzed, and the leakage amount under the corresponding values of different factors was also analyzed. The results indicated that increase in the lubricating oil temperature, viscosity, pressure ratio, and seal clearance lead to decrease in the sealing effect and increases in the leakage. And the effect of rotational speed on the seal is low. To verify numerical simulation, the results of numerical analysis, a test bench was set up to simulate the work of the reducer, and the sealing effect of the sealing structure was examined. The simulation result was compared with the actual results. Both the numerical simulation and experiment indicate that the labyrinth seal achieves the desired sealing effect.
\end{abstract}

\section{Introduction}

It is difficult to ensure the long-term operation of a contact seal owing to its contact friction and limited service life. However, a labyrinth seal is a type of noncontact seal with clearance or throttle seal. Direct contact between the rotating shaft and sealing tooth is absent, and the gap is small. Thus, there is no contact, wear, and need for lubrication. A gap exists between the rotating shaft and sealing teeth. This permits thermal expansion and the increase of operating speed of the machine. The labyrinth seal is a classical dynamic seal that is used in rail transit gear boxes, industrial gear boxes, and compressors $[1,2]$. A labyrinth seal implies that a certain number of throttling gears exist between moving parts and stationary parts leading to the formation of several twists and turns between the tooth and wall of the chamber. Hence, throttling effect and energy effect occur when the mediators go through the seal teeth and the seal chamber, thereby reducing the amount of leakage [3]. It is characterized by a high-pressure ratio, simple structure, and high reliability [4]. Thus, it is crucial to perform an in-depth investigation of the mechanism of labyrinth seal to design a reasonable labyrinth seal structure.
Given a host of in-depth studies in the labyrinth seal field, significant experience and improvement were gained globally in recent years. Kervistin [5] designed an apparatus for an engine that adjusts the labyrinth seal clearance and used the principles of thermal expansion and contraction. Yucel et al. [6] combined numerical and experimental investigations to analyze the main factors that affect the performance of the labyrinth seal including temperature and pressure differences, number of cavities, and tooth shape. Willey et al. [7] used the top of a turbine blade to replace the labyrinth seal tooth to seal the rotor, thereby breaking conventional the limitations of the labyrinth seal application and expanding its scope of applications.

Nowadays, numerical simulation has been proven as a promising technique since it can provide much information which cannot be measured via experimental tools $[8,9]$. In the labyrinth seal research method, computational fluid dynamics (CFD) is increasingly used to analyze the flow inside labyrinth seals $[10,11]$. Hodkinson [12] initially adopted a fluid mechanics method as opposed to the thermodynamics method to analyze the direct action of the fluid and used a special treatment of the outlet based on the nozzle model. Subsequently, Kearton and Keh [7] used the nozzle method 


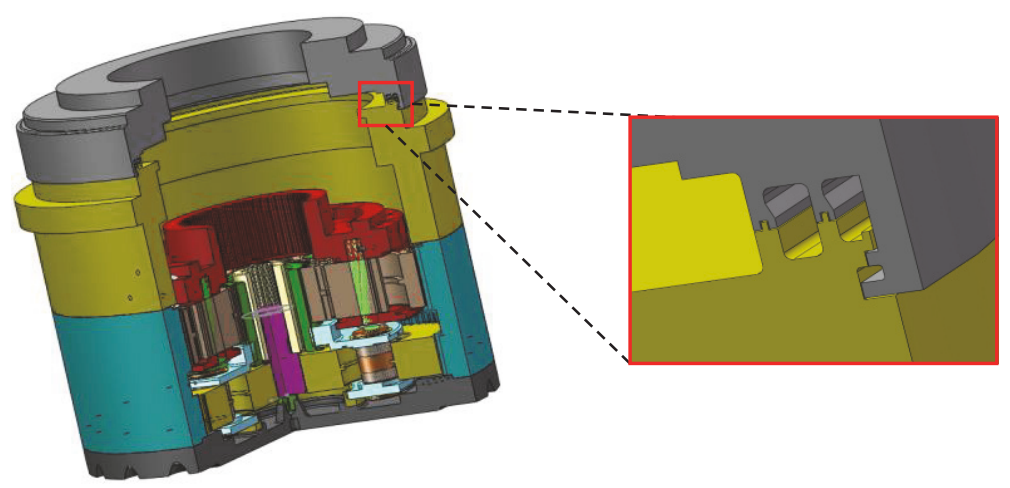

FIGURE 1: A labyrinth seal of hub reduction gear.

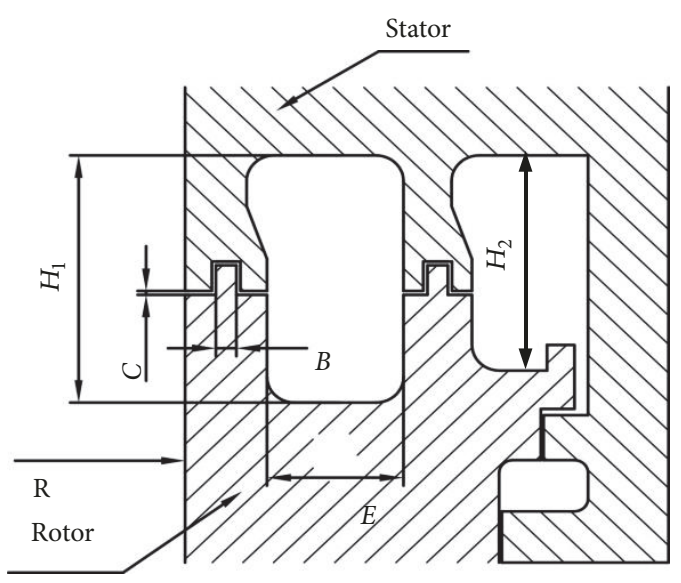

(a)

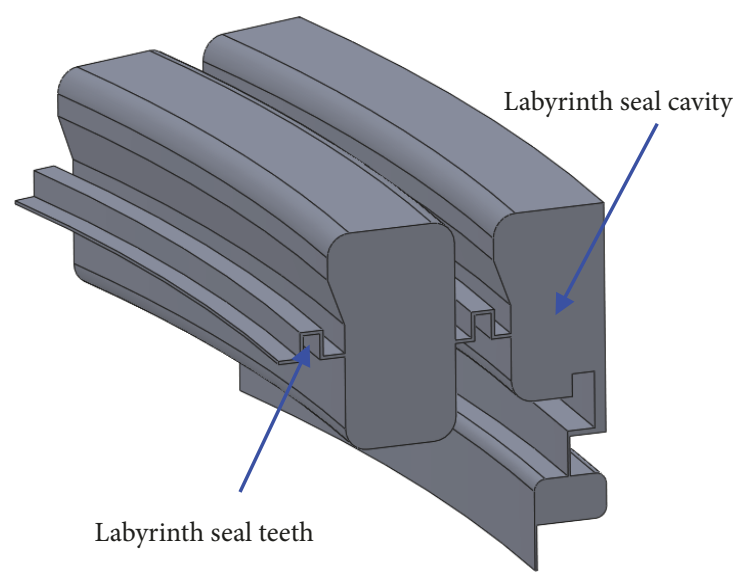

(b)

Figure 2: Schematic diagram of a labyrinth seal. (a) Two-dimensional section of labyrinth structure. (b) Three-dimensional flow model of labyrinth.

to simulate the flow at the seal outlet, although they used a reduced nozzle and considered blocking flow. While calculating the discharge coefficient, they also considered the factors corresponding to seal clearance and pressure ratio. $\mathrm{Wu}$ Te [13] considered the labyrinth seal at the small-gear motor end of the high-speed EMU drive gearbox as the research object and established a numerical simulation model of the labyrinth seal. He considered the effect of cavity depth on sealing performance, determined optimum cavity depth, and investigated the effect of pressure difference and rotational speed on leakage.

The study discusses the labyrinth seal structure that is designed for a wheel reducer. Taking the effect of the sealing structure into account, an analytical and computational model is established, and the flow characteristics of fluid in the labyrinth are analyzed via the CFD analysis method. The effects of temperature, inlet and outlet pressure, liquid viscosity, rotational speed of the rotor, and geometric size of the labyrinth on labyrinth sealing effect are investigated via the variable control method.

To verify the actual effect of the sealing structure, the sealing structure entity is manufactured, and a test bench is set up to perform the test. The sealing effect of the labyrinth seal structure is tested at different oil heights, speeds, and clearances.

\section{Structure of Labyrinth Seal}

The labyrinth seal is applied to the wheel-side reducer, as shown in Figure 1 which details the position and structure of a labyrinth seal.

\section{Calculation Method of Simulation}

3.1. Fluid Model Structure. The cross-section size of the flow field is shown in Figure 2(a), and the parameters are shown in Table 1. The sealing structure of the wheel-side reducer should conform to the actual situation. Thus, the rotational speed of the rotating part is designated in the range of $29.7-45.9 \mathrm{r} / \mathrm{min}$.

Under actual working conditions, the internal flow field of labyrinth seal comprising radial, axial, and circumferential turbulent flow is extremely complex. The authenticity of the results is affected if two-dimensional flow field analysis is used. Therefore, the 3D CFD method is used to model the labyrinth seal [14], as shown in Figure 2(b). The complete labyrinth model is large and the internal flow is symmetrical. Hence, 1/24 model of the flow field is selected to simulate the model to decrease calculation time and improve calculation efficiency. 


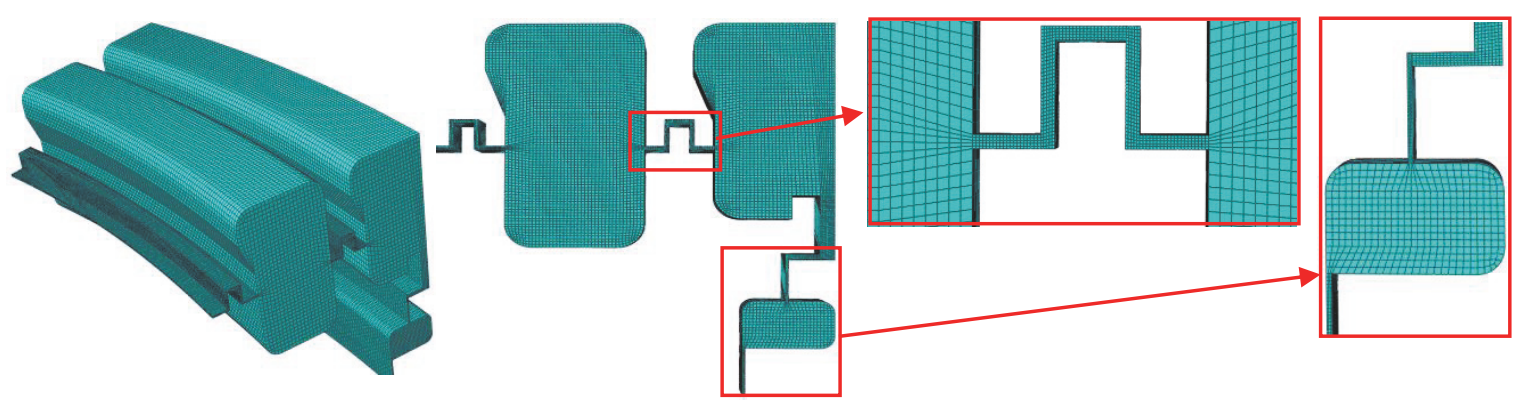

FIGURE 3: Mesh division of labyrinth fluid computation.

TABLE 1: Parameters of the labyrinth seal structure.

\begin{tabular}{lcccccc}
\hline Parameters & $H_{1} / \mathrm{mm}$ & $H_{2} / \mathrm{mm}$ & $C / \mathrm{mm}$ & $B / \mathrm{mm}$ & $E / \mathrm{mm}$ & $R / \mathrm{mm}$ \\
\hline Value & 13 & 11 & 0.3 & 1.5 & 8 & 170 \\
\hline
\end{tabular}

3.2. Mesh Generation. The computational region needs to be meshed to examine the flow field inside the labyrinth seal. To achieve computational accuracy and decrease computational time, the model is structured in several blocks and meshes are refined in special regions. The results of the meshing are shown in Figure 3.

3.3. Condition Setting and Calculation. The internal flow of the labyrinth is described by the governing equation of energy-conserving fluid. In the study, we reference common boundary condition types that are widely used for seal models [15-17] and adopt the following assumptions to simplify initialization parameters.

(1) The working medium in the flow field corresponds to liquid. In addition to analyzing the effect factors of viscosity, all other calculation models use water as the working medium.

(2) The boundary condition of the wall is set as the adiabatic boundary.

(3) The flow inside the labyrinth corresponds to turbulent flow.

(4) The radial and axial displacements of the rotor are ignored in the numerical simulation because of the high symmetry of the structure.

The outlet pressure corresponds to $1 \mathrm{~atm}$. Inlet pressure is 1.4 times the outlet pressure and is equal to $141855 \mathrm{~Pa}$. Adiabatic wall and the fluid medium correspond to water, we use $\mathrm{k}-\varepsilon$ two-equation turbulence model, and the continuity equation is discretized by a second-order upwind scheme and solved via a coupled implicit solver.

The residual values of continuity equation, directional velocity, turbulent kinetic energy $k$, and dissipation rate are less than $10^{-3}$, and the difference between inlet and outlet flow rate is less than $0.1 \%$. In the calculation process, the residual error in every direction infinitely approaches the convergence standard as shown in Figure 4.

We set the inlet pressure $P_{0}$ as $141855 \mathrm{~Pa}$, outlet pressure $P_{n}$ as $101325 \mathrm{~Pa}$, and inlet temperature as $313 \mathrm{~K}$ with an adiabatic wall and liquid medium as Lubricating oil. The calculation obtains the pressure field of the labyrinth, as shown in Figure 5.

The rationality of the calculation results and the principle of the labyrinth seal are verified by the velocity cloud chart and velocity vector diagram of the labyrinth flow field. The results are shown in Figures 6 and 7.

\section{Simulation Results and Analysis}

The study mainly explores the optimal size of the labyrinth seal with decrease in the leakage and also analyzes the effects of viscosity and temperature, pressure ratio, and rotational speed on the leakage.

4.1. Effects of Viscosity and Temperature on Leakage Rate. The calculation model is the same as that in a labyrinth seal of hub reduction gear. The fluid medium is varied. Five brands of lubricating oil are used for the analysis. The temperature range is set as $10-60^{\circ} \mathrm{C}$. The used parameters are shown in Table 2 .

In the analysis, the variation curves of viscosity and temperature on leakage rate are obtained, as shown in Figure 8.

As shown in Figure 8, the viscosity of lubricating oil decreases with increases in the working temperature (from $10^{\circ} \mathrm{C}$ to $60^{\circ} \mathrm{C}$ ) decreases in the lubricating oil grade (from VG100 to VG22) leakage increases. Therefore, the operation of the transmission device should select grease with higher viscosity and avoid higher working temperature.

4.2. Effects of Pressure Ratio and Speed on Leakage Rate. The calculation model is the same as that in a labyrinth seal of hub reduction gear. The pressure ratio and speed vary. The outlet pressure is $101325 \mathrm{~Pa}$, the pressure ratio ranges from 1.2 to 2.0 , and the rotational speed correspond to $200 \mathrm{r} / \mathrm{min}, 300 \mathrm{r} / \mathrm{min}$, $400 \mathrm{r} / \mathrm{min}$, and $500 \mathrm{r} / \mathrm{min}$. The data are shown in Table 3.

The values of leakage under different speeds and pressure ratios are obtained via numerical analysis, as shown in Figure 9. 


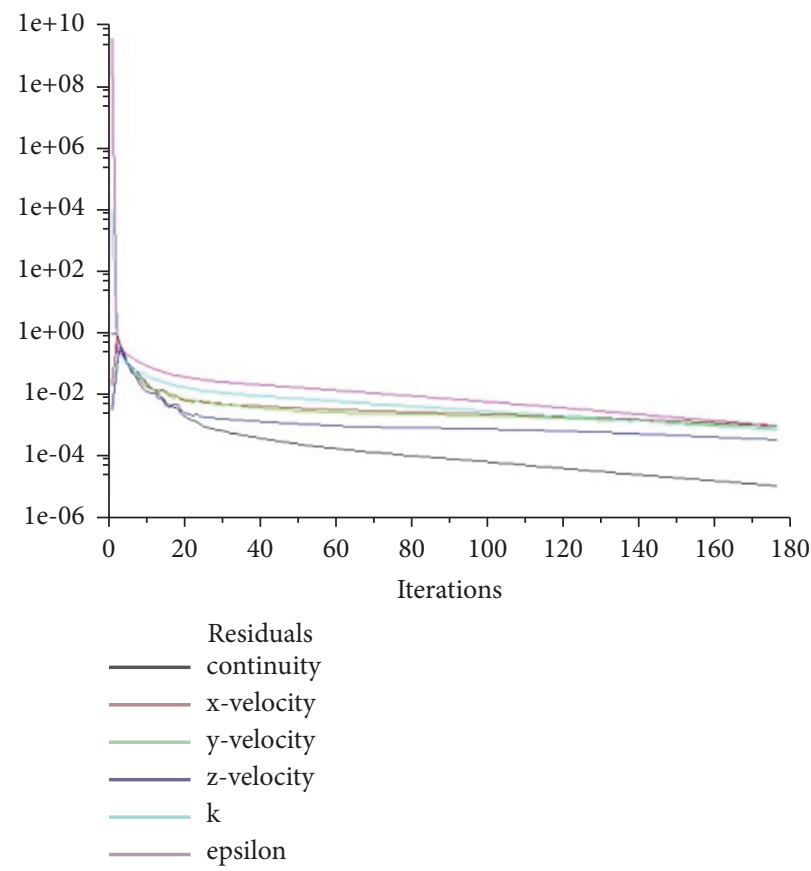

FIgURE 4: Residual curve.
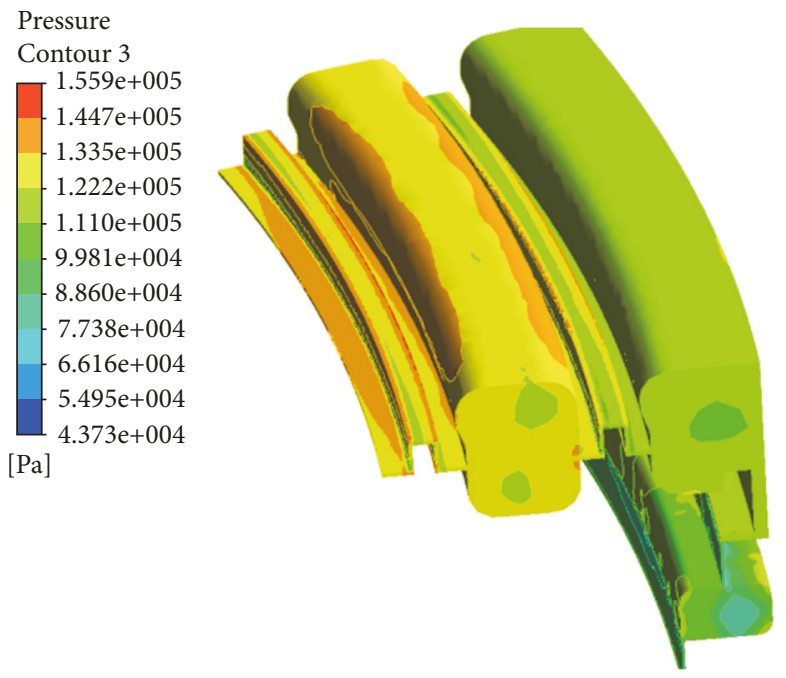

Figure 5: Pressure field cloud map of the three-dimensional flow field in the labyrinth.

TABLE 2: Leakage values (kg/s) of different lubricant brands with varying viscosity and temperature.

\begin{tabular}{lcccccc}
\hline Lubricating oil brands & \multicolumn{5}{c}{ Temperature $\left({ }^{\circ} \mathrm{C}\right)$} \\
& 10 & 20 & 30 & 40 & 50 & 0.01825 \\
\hline VG22 & 0.00404 & 0.00728 & 0.01250 & 0.02435 & 0.03036 \\
VG32 & 0.00227 & 0.00363 & 0.00681 & 0.01259 & 0.01847 \\
VG46 & 0.00193 & 0.00297 & 0.00466 & 0.00978 & 0.01308 & 0.02587 \\
VG68 & 0.00150 & 0.00208 & 0.00340 & 0.00525 & 0.00966 & 0.01861 \\
VG100 & 0.00128 & 0.00176 & 0.00240 & 0.00371 & 0.00587 & 0.00945 \\
\hline
\end{tabular}


Velocity

Contour 2

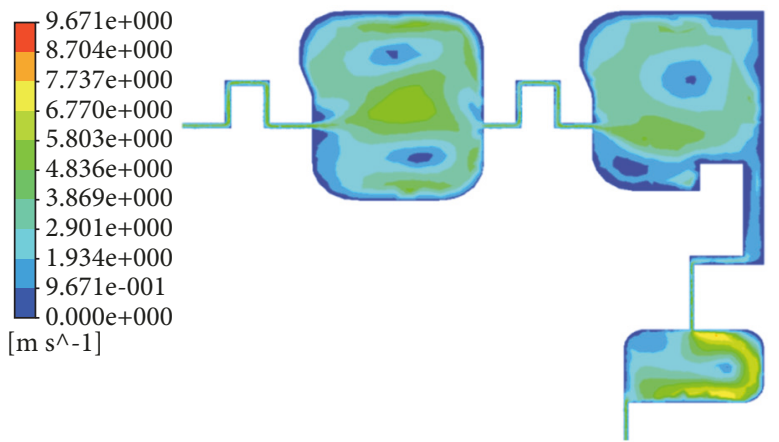

FIGURE 6: Velocity cloud map of the labyrinth flow field.

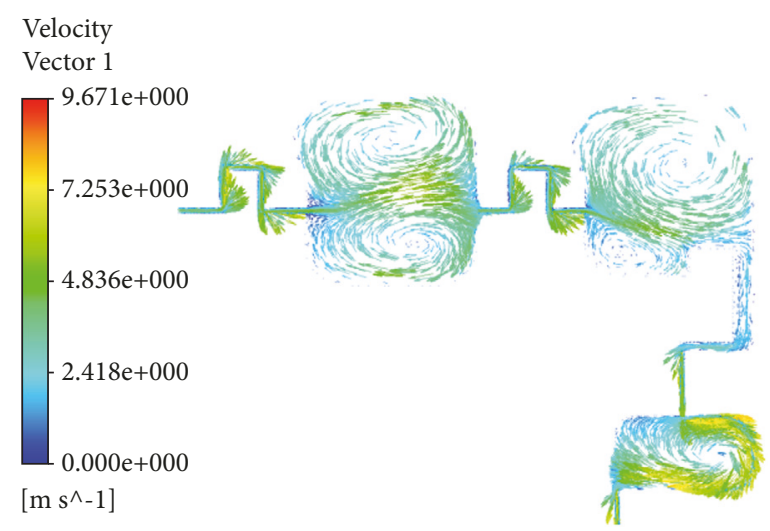

FIGURE 7: Velocity vector diagram of the labyrinth flow field.

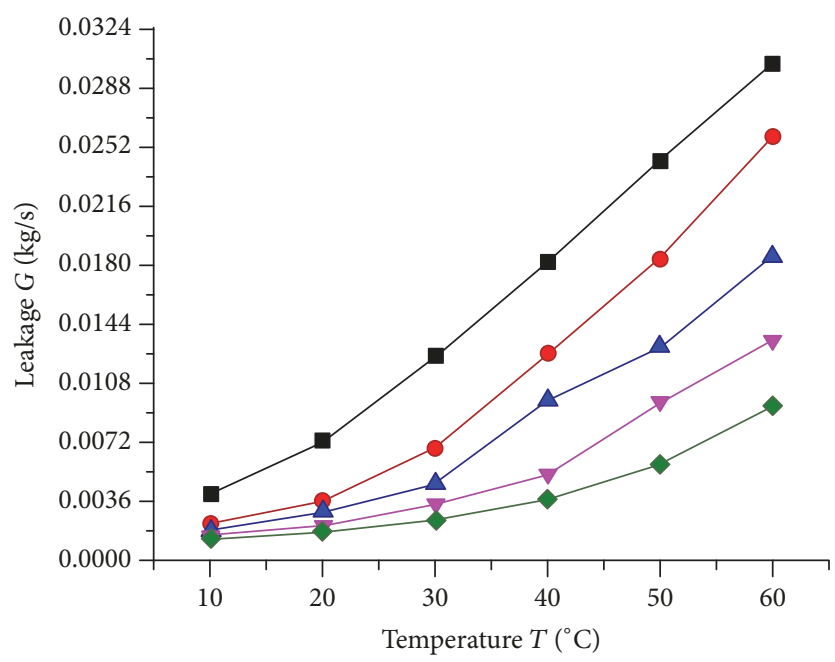

Lubricant brands

$$
\begin{array}{ll}
\longrightarrow & \text { VG22 } \\
- \text { VG32 } \\
- \text { VG46 } \\
\longrightarrow \text { VG68 } \\
\square \text { VG100 }
\end{array}
$$

FiguRE 8: Variation in the leakage rate relates to the temperature for different viscosity values. 


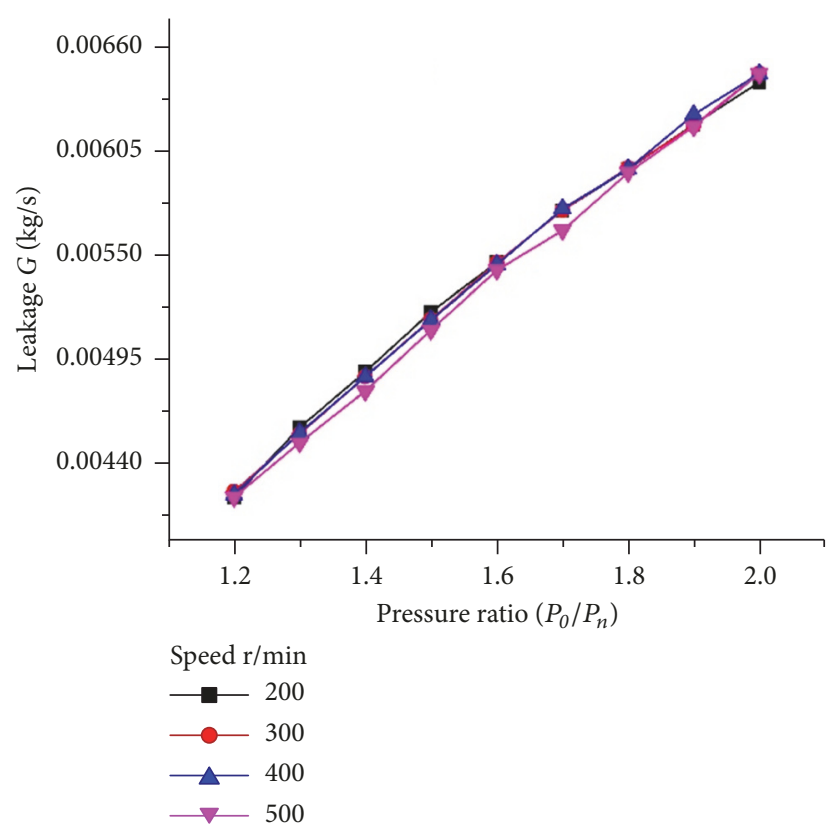

FIGURE 9: Variation in the leakage rate relative to the pressure ratio for different speeds.

TABLE 3: Leakage value $(\mathrm{kg} / \mathrm{s})$ under different speeds and pressure ratios.

\begin{tabular}{|c|c|c|c|c|c|}
\hline \multirow{2}{*}{ Working conditions } & & \multicolumn{4}{|c|}{ Speed $(\mathrm{r} / \mathrm{min})$} \\
\hline & & 200 & 300 & 400 & 500 \\
\hline \multirow{9}{*}{ Pressure ratio $\left(P_{0} / P_{n}\right)$} & 1.2 & 0.004226 & 0.004256 & 0.004241 & 0.004227 \\
\hline & 1.3 & 0.004596 & 0.004560 & 0.004566 & 0.004515 \\
\hline & 1.4 & 0.004889 & 0.004863 & 0.004862 & 0.004787 \\
\hline & 1.5 & 0.005204 & 0.005166 & 0.005162 & 0.005109 \\
\hline & 1.6 & 0.005465 & 0.005464 & 0.005453 & 0.005424 \\
\hline & 1.7 & 0.005739 & 0.005740 & 0.005748 & 0.005634 \\
\hline & 1.8 & 0.005958 & 0.005964 & 0.005958 & 0.005939 \\
\hline & 1.9 & 0.006194 & 0.006188 & 0.006245 & 0.006183 \\
\hline & 2.0 & 0.006417 & 0.006463 & 0.006461 & 0.006462 \\
\hline
\end{tabular}

Pressure ratio and rotational speed are two important factors that affect leakage. The results indicate that increases in the pressure ratio at both ends of straight-through labyrinth seal lead to increases in the leakage $[18,19]$. When the rotational speed is lower than a certain value in a low speed range, it is generally believed that the effect of rotational speed on the leakage of labyrinth seal is extremely low. When the rotational speed exceeds the value, increases in the rotational speed will decrease the leakage of the labyrinth seal to a certain extent, although the effect is extremely low and does not exceed $4 \%$ of the overall sealing, as verified by the numerical simulation.

4.3. Effect of Labyrinth Clearance Magnitude to the Leakage Rate. The calculation model is the same as that in a labyrinth seal of hub reduction gear, except for the fact that the axial clearance $C$ ranges from $0.15 \mathrm{~mm}$ to $0.95 \mathrm{~mm}$. The leakage rate under different pressure ratios is calculated, and the change in leakage rate with the sealing gap is observed. As shown in Figure 10, the leakage increases with increasing the advance of clearance when the pressure ratio is fixed. This is because increases in the clearance decrease the throttling effect when fluid passes the clearance, vortex weakens after fluid enters the cavity, and energy dissipation decreases. Additionally, the leakage exhibits the same trend under different pressure ratios and the slope of the curve increases with increases in the pressure ratio. The reason for the phenomenon is that the fluid velocity flowing into the cavity increases and the vortex expands. Hence, the leakage value increases with the advance of the pressure ratio. This shows that labyrinth clearance magnitude significantly affects leakage. A lower clearance should be selected if permitted by processing costs and easy installation conditions [12].

4.4. Effect of Cavity Depth to the Leakage Rate. Except for the fact that the cavity depth $H_{1}$ ranges from $4.3 \mathrm{~mm}$ to 19.3 $\mathrm{mm}$ and $\mathrm{H}_{2}$ ranges from $4.3 \mathrm{~mm}$ to $16.8 \mathrm{~mm}$, the calculation model is the same as that in a labyrinth seal of hub reduction gear. Figure 11 shows the four types of pressure distribution at different cavity depths. Evidently, the pressure changes 


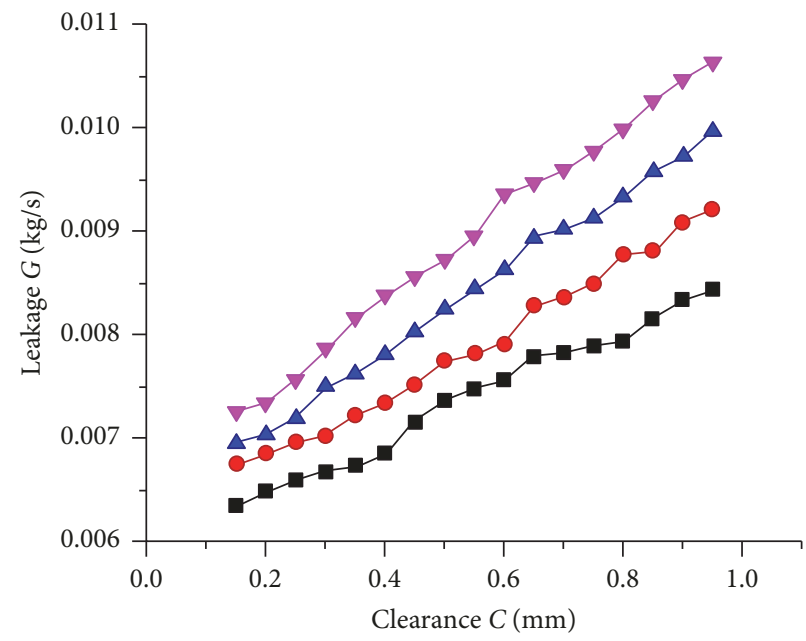

Pressure ratio

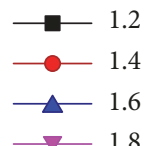

FIGURE 10: Change of leakage with clearance under different pressure ratios.

Pressure

Contour 2

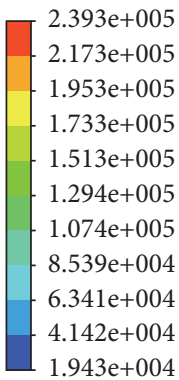

[Pa]

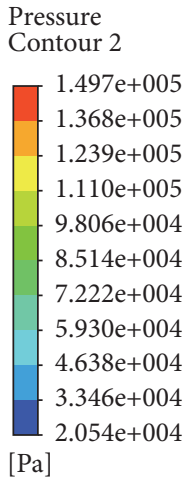

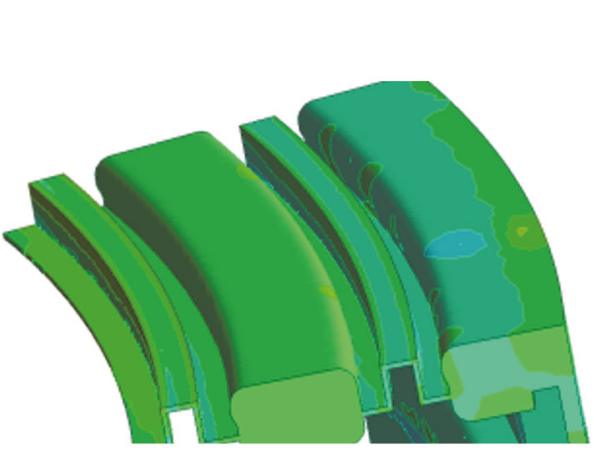

(a) $H_{1}=4.3 \mathrm{~mm}, H_{2}=4.3 \mathrm{~mm}$

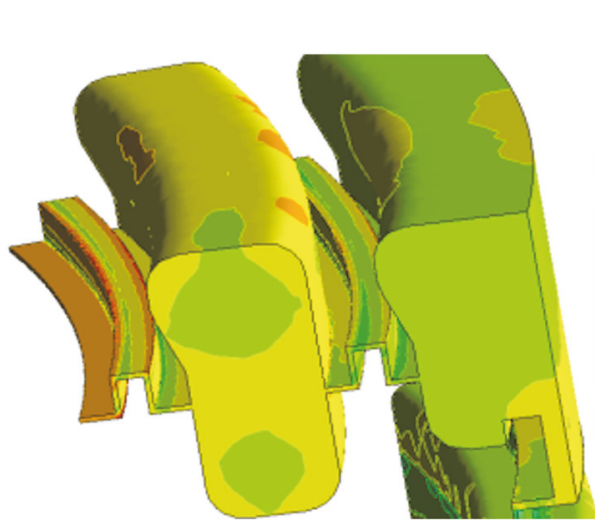

(c) $H_{1}=16.3 \mathrm{~mm}, H_{2}=13.8 \mathrm{~mm}$

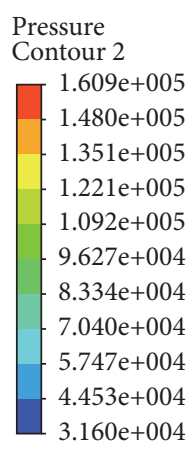

$[\mathrm{Pa}]$

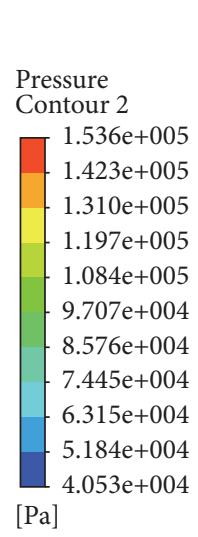

(b) $H_{1}=10.3 \mathrm{~mm}, H_{2}=9.8 \mathrm{~mm}$

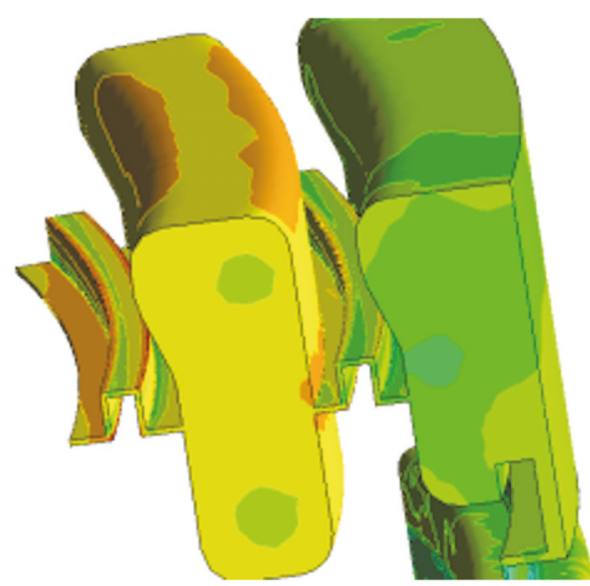

(d) $H_{1}=19.3 \mathrm{~mm}, H_{2}=16.8 \mathrm{~mm}$

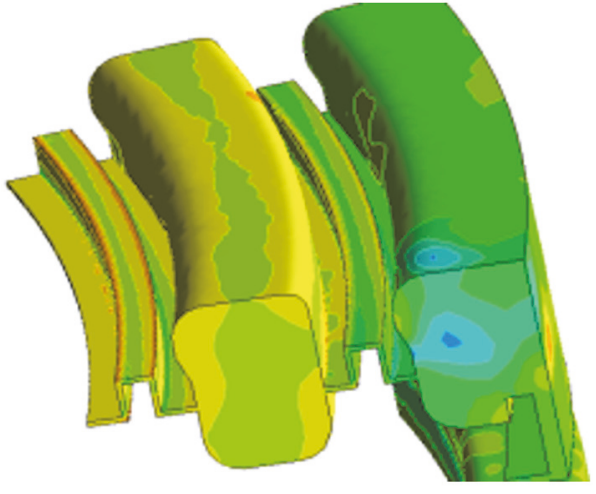

FIGURE 11: Pressure cloud chart with different cavity depth values. 


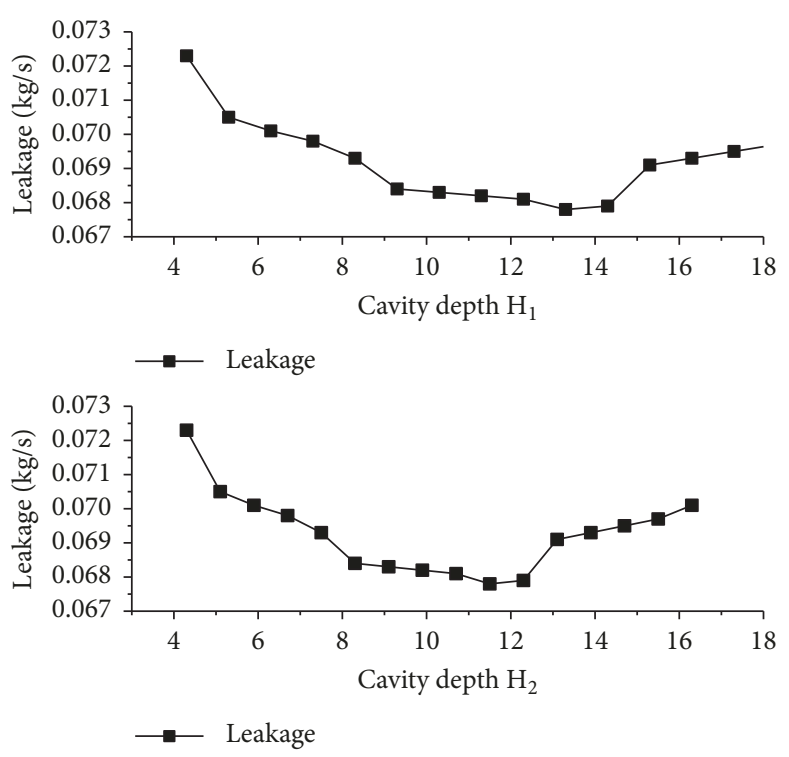

FIGURE 12: Plot of leakage with different cavity depths.

with increases in the cavity depth. The vortex is not evident in Figure 11(a), and it is evident in Figures 11(b) and 11(c). However, in Figure 11(d), the vortex only happens in a small part. This reveals that cavity depth promotes the formation of vortices within a certain range, and the cavity fluid vortex weakens when cavity depth increases constantly and results in decreases in energy dissipation.

Figure 12 shows the leakage curve with different cavity depths. It reveals that leakage decreases rapidly when the cavity depth increases from $4.3 \mathrm{~mm}$ to $6.0 \mathrm{~mm}$, and the leakage decreases slowly when the depth continues to increase. Specifically, the $H_{1}$ value increases from $10 \mathrm{~mm}$ to $14 \mathrm{~mm}$, the $\mathrm{H}_{2}$ value increases from $8 \mathrm{~mm}$ to $12 \mathrm{~mm}$, and the leakage stabilized at approximately $0.068 \mathrm{~kg} / \mathrm{s}$, while the leakage increases as the cavity depth continues to increase. It is concluded that increases in the cavity depth effectively decrease the leakage within a certain range. In the actual working conditions, there is an optimum size range of cavity depth that effectively decreases leakage to satisfy sealing requirements and the production process requirement.

4.5. Effects of Cavity Width on the Leakage Rate. The calculation model is the same as Figure 2, and only the cavity width $E$ ranges from $2 \mathrm{~mm}$ to $18 \mathrm{~mm}$. Figure 13 shows the velocity contour and velocity vector under different cavity widths. As shown in Figure 13(a), the cavity width is extremely narrow, there are four effective vortexes that drive fluid flowing in the cavity, and kinetic energy is converted to thermal energy. As shown in Figure 13(b), there are two vortexes in cavity that form the contraction in the cavity outlet, and the fluid generates intense friction in the cavity during the process. There is no evident vortex in the cavity in Figure 13(c), and the sealing effect weakens.

Figure 14 shows the curve of leakage with different cavity widths. It reveals that leakage increases slowly when the cavity width increases from $2 \mathrm{~mm}$ to $10 \mathrm{~mm}$. The slope of the curve increases rapidly when the cavity width continues increasing. The increase in cavity width almost does not affect the leakage within a certain range. However, leakage increases when the cavity width continues expanding to a certain value.

There is a certain width value below which the decrease in the width weakens the leakage. However, the smaller size makes the process and installation more difficult. Therefore, rational design of the cavity width dimension should be based on the effect of cavity width on leakage in actual working conditions such that it satisfies sealing requirements and the production process requirements.

4.6. Effects of the Ratio of Depth to Width on the Leakage Rate. This section numerically analyzes the effect of the ratio of depth to width on the leakage rate, as shown in Figure 15. The leakage exhibits the same changing trend and increases in the ratio effectively induce the decrease of leakage within a certain range. Leakage increases if the ratio exceeds a certain value and continues expanding, as shown in Figures 15(a), 15(b), and 15(c). The ratio of depth to width exhibits different effects on leakage under different cavity widths. A lower leakage is observed in the range of 1.1 to 3.0 when the width corresponds to $4 \mathrm{~mm}$, and the range of optimum ratio decreases with increases in the width. There is an optimum ratio of depth to width of about 1.1 in actual working conditions. The effect of the ratio on leakage increases with the advance of width.

\section{Experimental Verification}

The above sections analyzed the theoretical calculation method of leakage of the labyrinth seal and used a numerical simulation analysis method to examine the mechanism of the labyrinth seal and provide an important basis for designing the labyrinth seal of the gearbox. The internal flow field is 


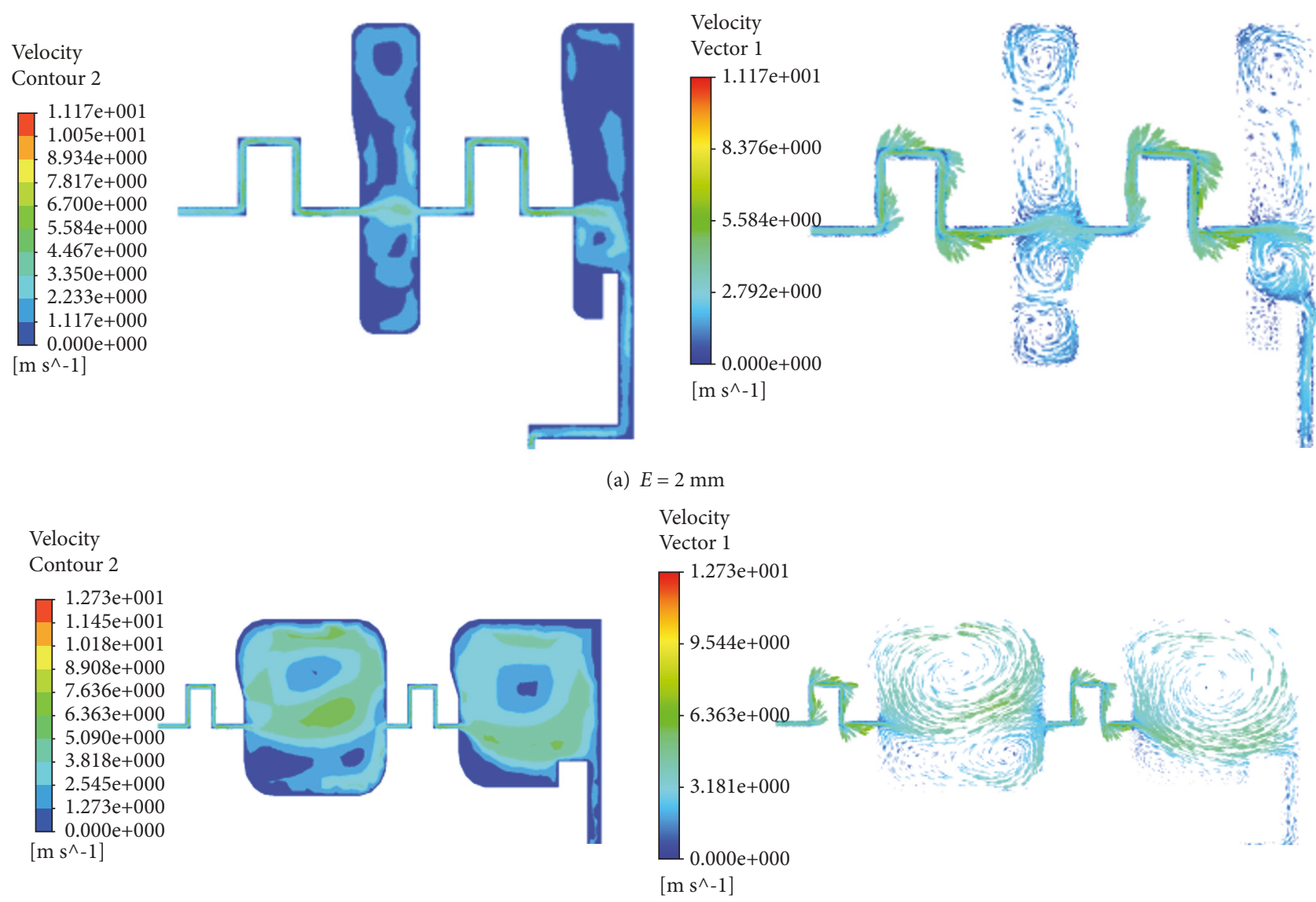

(b) $E=10 \mathrm{~mm}$
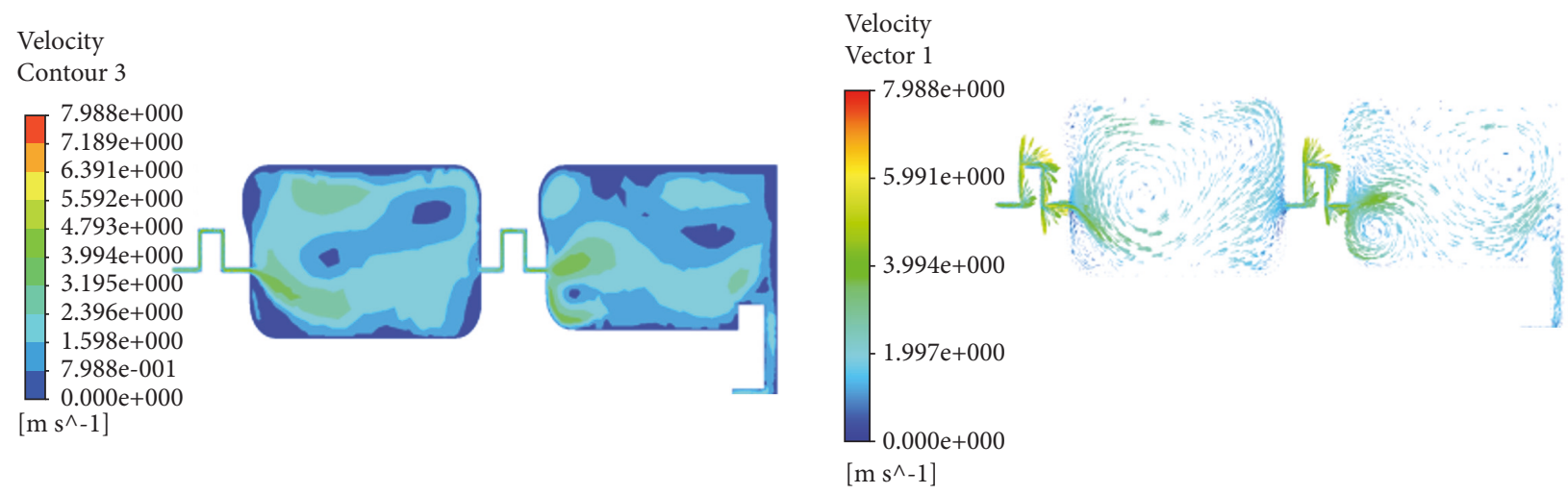

(c) $E=18 \mathrm{~mm}$

FIGURE 13: Velocity contour and velocity vector under different cavity widths.

extremely complex with the exception of the simulation, and thus experimental verification is required.

5.1. Structure Design of the Labyrinth Seal. The experiment is based on factors of leakage that are designed based on the numerical calculation. The numerical results indicate that decreases in the clearance lead to improvement in the sealing performance. However, an excessively low clearance leads to collision, structural abrasion, and even sealing failure if the machining accuracy is not extremely high. Thus, we attempt to select a smaller clearance under the condition of meeting processing costs and installation requirements. This is based on the following empirical expression:

$$
C=0.2+\frac{(0.3 \sim 0.6) d}{1000}
$$

Rotor diameter $d$ corresponds to $340 \mathrm{~mm}$, and calculated clearance $C$ corresponds to $0.3 \mathrm{~mm}$. Based on the numerical calculation results, cavity depth $H_{1}$ value corresponds to 13 


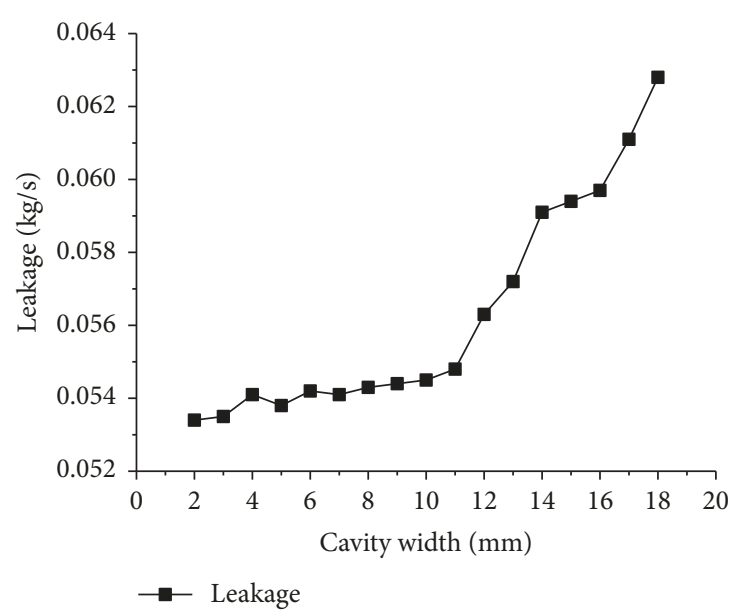

FIGURE 14: Graph of leakage with respect to the cavity width.
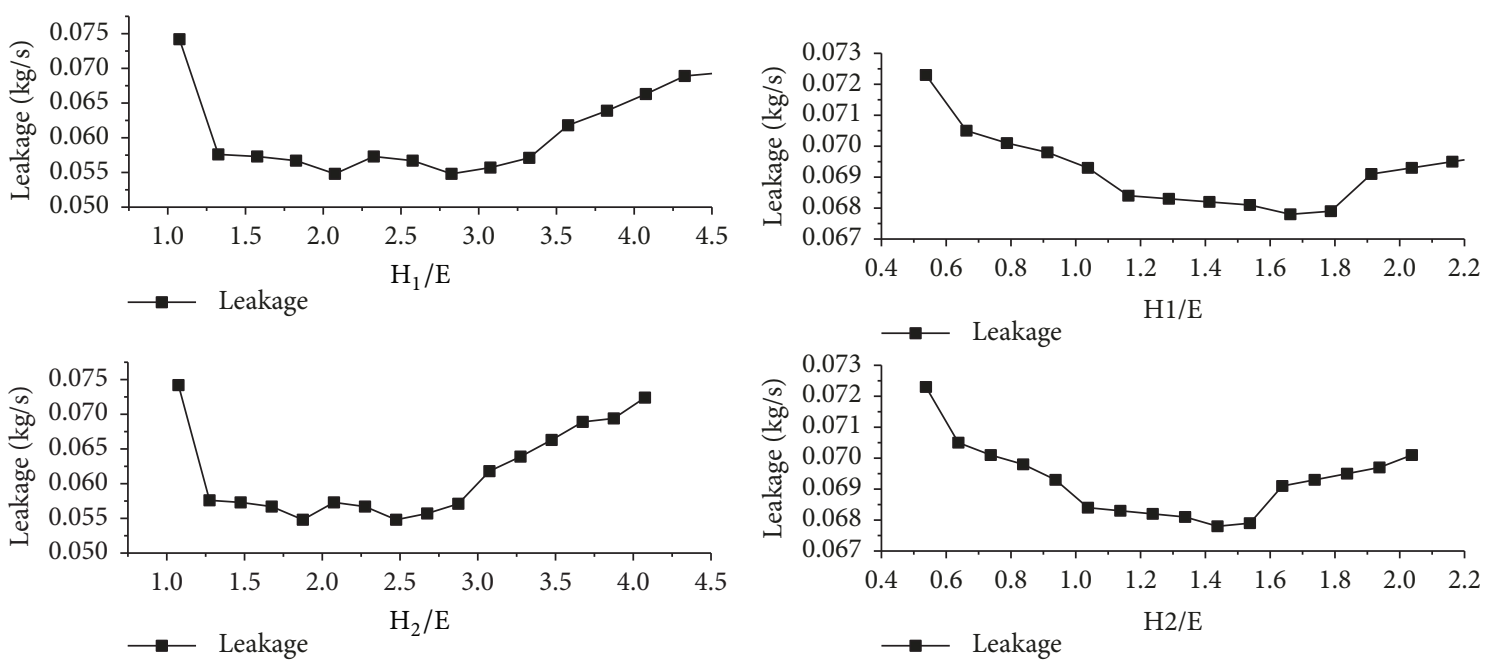

(a) $E=4$

(b) $E=8$
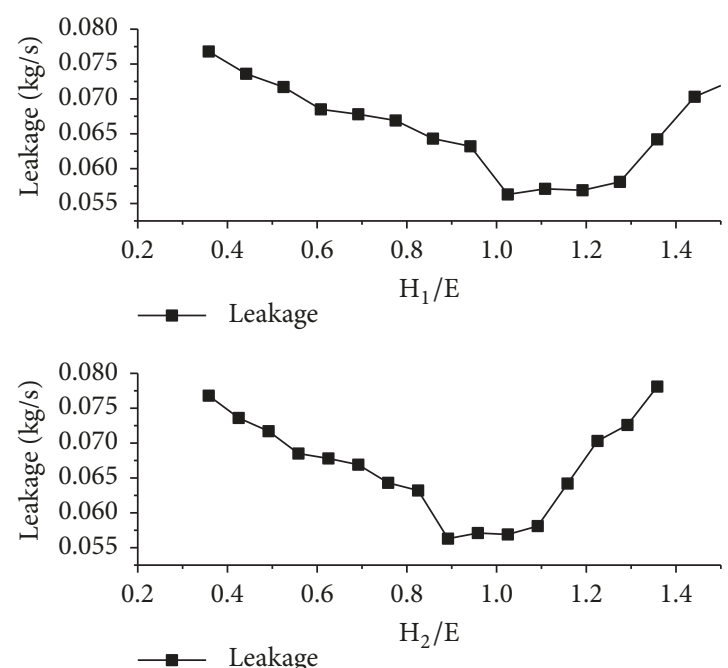

(c) $E=12$

FIGURE 15: Plot of leakage with respect to the ratio of depth to width. 
TABLE 4: Test record with different liquid levels.

\begin{tabular}{|c|c|c|c|}
\hline Liquid level $/ \mathrm{mm}$ & Leakage & Liquid level $/ \mathrm{mm}$ & Leakage \\
\hline 5 & No oil leaking & 40 & No oil leaking \\
\hline 10 & No oil leaking & 45 & $\begin{array}{l}\text { Leaking in stationary state } \\
\text { No leaking in running state }\end{array}$ \\
\hline 15 & No oil leaking & 50 & $\begin{array}{l}\text { Leaking in stationary state } \\
\text { No leaking in running state }\end{array}$ \\
\hline 20 & No oil leaking & 55 & $\begin{array}{l}\text { Leaking in stationary state } \\
\text { No leaking in running state }\end{array}$ \\
\hline 25 & No oil leaking & 60 & $\begin{array}{l}\text { Leaking in stationary state } \\
\text { No leaking in running state }\end{array}$ \\
\hline 30 & No oil leaking & 65 & $\begin{array}{l}\text { Leaking in stationary state } \\
\text { No leaking in running state }\end{array}$ \\
\hline 35 & No oil leaking & & \\
\hline
\end{tabular}

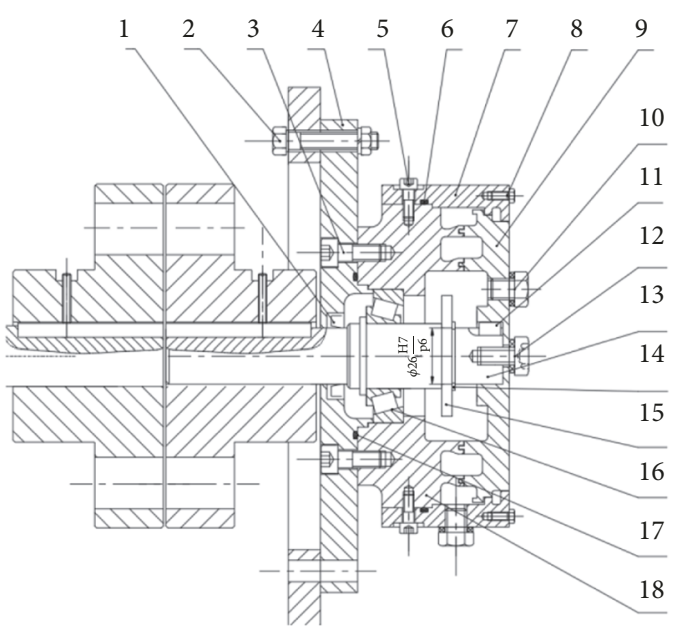

FIGURE 16: Structure diagram of labyrinth seal test-bed. 1: lip-type packing; 2: bolt; 3 : screw-a; 4 : input shaft end cover; 5 : screw-b; 6 : O-type ring-a; 7: shell; 8: screw-c; 9: sealing element-a; 10: oil plug; 11: bond; 12: screw-d; 13: shaft; 14: circlips for shaft; 15: thrower; 16: taper roller bearing; 17: O-type ring-b; 18: sealing element-b.

$\mathrm{mm}, H_{2}$ value corresponds to $11 \mathrm{~mm}$, and cavity width $E$ value corresponds to $10 \mathrm{~mm}$.

5.2. Test Device. Based on the design shape of labyrinth seal, a few parts in Figure 13 are processed, and the test rig is assembled. Figure 16 shows the structure diagram of the testbed.

The physical structure of the test-bed is shown in Figure 17. A layer of white paint is coated on the surface of the seal prior to the test to clearly observe the leakage. This can be observed in the test pictures shown in Figure 18.

5.3. Effects of Lubrication Oil Levels on Leakage Rate. The volume of lubricant required for different liquid level fillings is calculated; the relevant volume of lubricant is then injected, and liquid height is selected as shown in Figure 19. We control the rotation speed increasing from 0 to $300 \mathrm{rpm}$. The test is

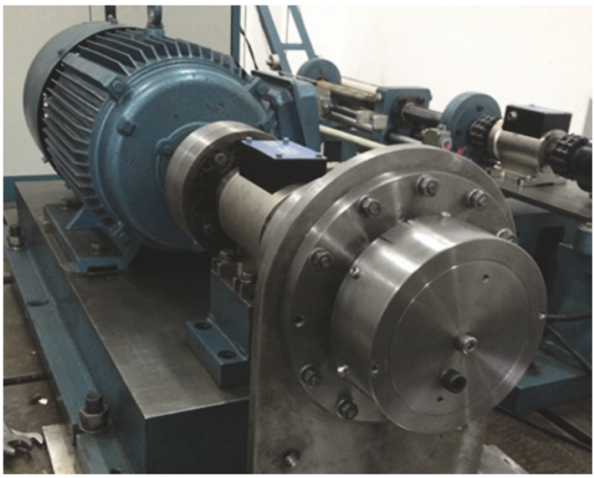

FIGURE 17: Sealing test bench.

completed, and the data is recorded, as shown in Figure 20 and Table 4.

The results indicate that when the liquid level reaches 45 $\mathrm{mm}$, the sealing surface begins to leak while the labyrinth seal is in the stationary state. The leakage increases when the liquid level continues increasing. Additionally, oil leakage is absent in the running state. This is because in the stationary state, increases in the liquid level increase cavity pressure. Furthermore, increases in the pressure increase the leakage. In the running state, a throttling effect is observed on the seal tooth interstice, and the pressure decreases when the fluid passes through the labyrinth seal, and thus the leakage decreases.

5.4. Effects of Rotation Speed on Leakage Rate. In order to investigate the effect of rotation speed on leakage rate, the experiment was done with the motor speed increasing from $30 \mathrm{rpm}$ to $300 \mathrm{rpm}$. The liquid level is $35 \mathrm{~mm}$ and the other conditions are constant. Data are observed and recorded every thirty minutes. The results are shown in Table 5. No leak was found. The seal does not change with increases in the motor speed. The consistence of experiment and numerical simulation conclusions verified the accuracy of the numerical simulation method. 

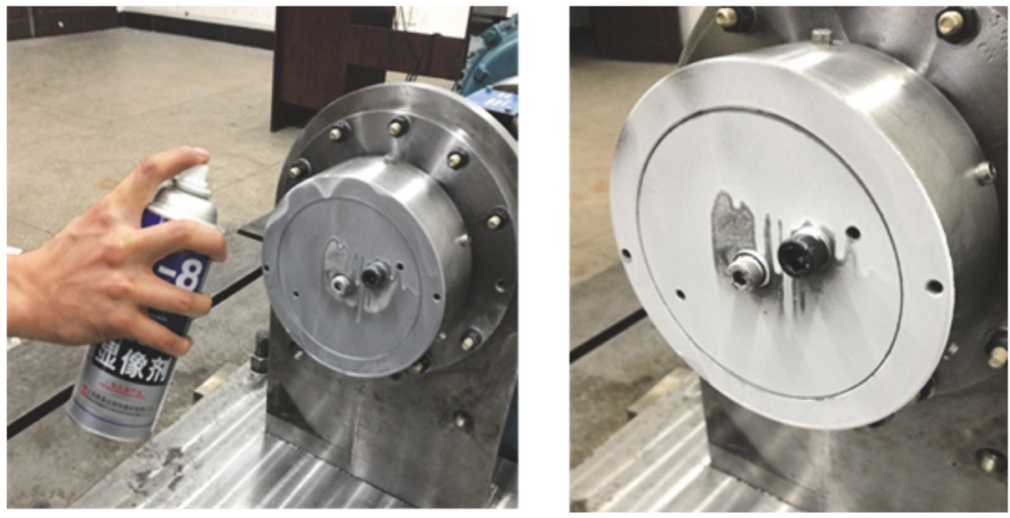

FIGURE 18: Spray developer.

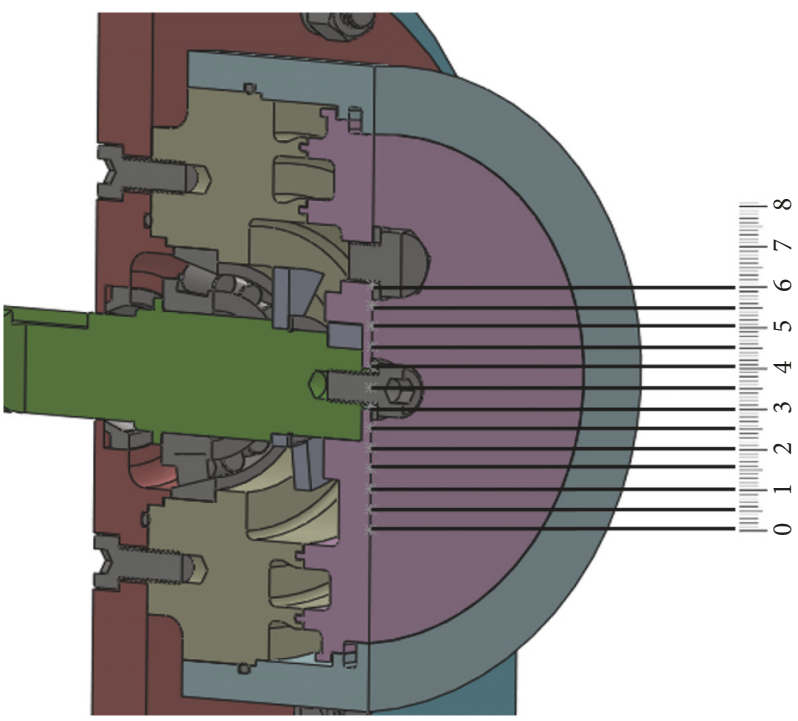

FIGURE 19: Schematic diagram of the liquid level.

TABLE 5: Test record with different motor speeds.

\begin{tabular}{lcc}
\hline Time $(\min )$ & Speed $(\mathrm{r} / \mathrm{min})$ & Leakage \\
\hline 30 & 40 & No oil leaking \\
60 & 80 & No oil leaking \\
90 & 120 & No oil leaking \\
120 & 160 & No oil leaking \\
150 & 200 & No oil leaking \\
180 & 240 & No oil leaking \\
210 & 280 & No oil leaking \\
240 & 300 & No oil leaking \\
\hline
\end{tabular}

5.5. Effects of Labyrinth Clearance. In order to examine the influence of clearance on leakage, the motor speed is maintained at $40 \mathrm{rpm}$ and the clearance is adjusted from $0 \mathrm{~mm}$ to $0.8 \mathrm{~mm}$ by the spacer in the connection of the sealing element-a with the shaft. The result is shown in Figure 21 and Table 6 . It reveals that the leakage rate increases with increases in the clearance. The experiment results are consistent with the numerical simulation. This proves that the structure is reasonable and the numerical simulation is accurate.

\section{Conclusions}

The following conclusions are obtained:

(1) The simulation results confirmed that the reduction in the viscosity of the lubricant oil (such as elevating temperature and reducing lubricant oil grade) led to the increase in leakage, the pressure ratio significantly affected leakage, and they were positively related. When the speed was lower than a certain value in a low speed range, the effect of rotation speed was extremely weak.

(2) Cavity depth can effectively decrease the leakage rate in certain range, width effect is not obvious. The numerical simulation showed that there was an optimum ratio of depth to width of about 1.1 .

(3) The experiment results indicated that in a stationary state, the leakage exacerbated when the liquid level continued 


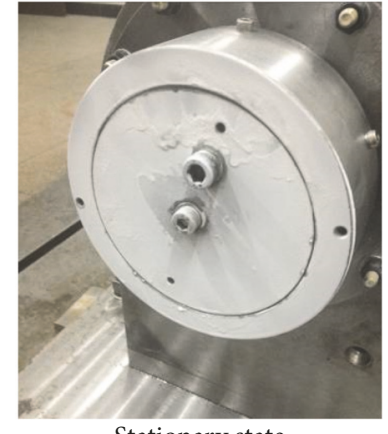

Stationary state

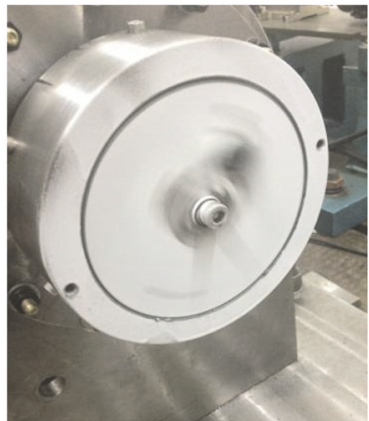

Running state

(a) Liquid level is $30 \mathrm{~mm}$

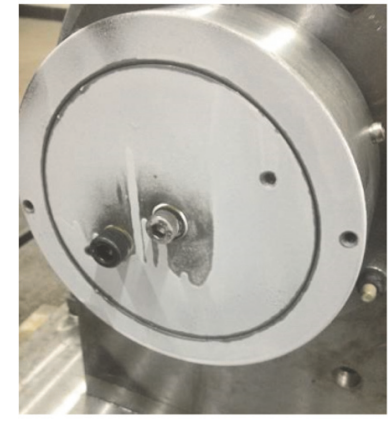

Stationary state

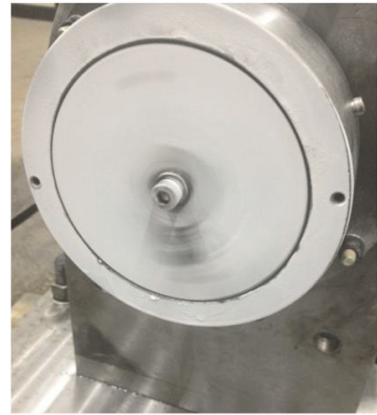

Running state

(b) Liquid level is $60 \mathrm{~mm}$

FIGURE 20: Condition of the seal with different liquid levels.

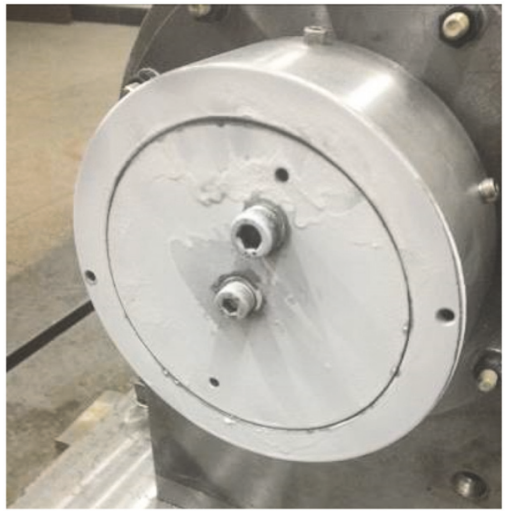

(a) $0.3 \mathrm{~mm}$

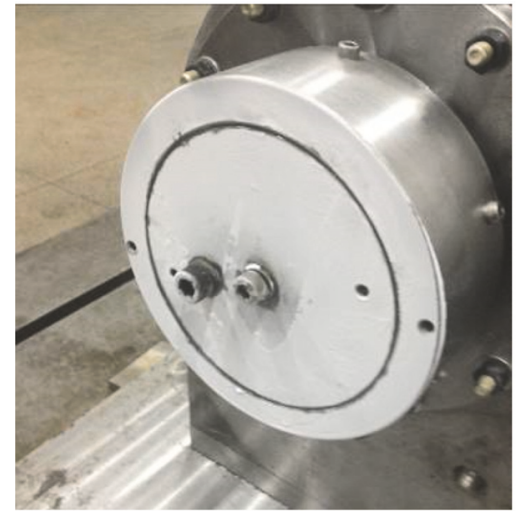

(b) $0.5 \mathrm{~mm}$

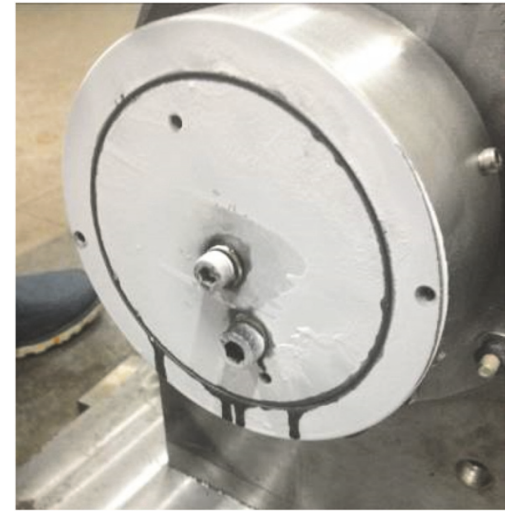

(c) $0.8 \mathrm{~mm}$

FIGURE 21: Effects of sealing with different clearance values.

TABLE 6: Test record with different clearance values.

\begin{tabular}{lcccccc}
\hline Clearance $/ \mathrm{mm}$ & 0.3 & 0.4 & 0.5 & 0.6 & 0.7 & 0.8 \\
\hline Leakage & No oil leaking & No oil leaking & A little oil marks & A little oil marks & Obvious oil marks & Oil drop \\
\hline
\end{tabular}

increasing after it reached a certain value and, under that value, there is no leakage. In the running state, there was no leakage when the liquid level or motor speed increased.

\section{Data Availability}

The structure size data used to support the findings of this study are currently under embargo while the research findings are commercialized. Requests for data will be considered by the corresponding author.

\section{Conflicts of Interest}

The authors declare that there is no conflict of interest regarding the publication of this paper.

\section{Acknowledgments}

This research was funded by Natural Science Foundation of Hunan (grant number 2018JJ4006) and the National
Independent Innovation Demonstration Area Foundation of Changsha Zhuzhou Xiangtan (grant number 2018XK2302).

\section{References}

[1] B. Lakshminarayana, Fluid Dynamics and Heat Transfer of Turbomachinery, Wiley, New York, NY, USA, 1996.

[2] R. E. Chupp, R. C. Hendricks, S. B. Lattime, and B. M. Steinetz, "Sealing in turbomachinery," Journal of Propulsion and Power, vol. 22, no. 2, pp. 313-349, 2006.

[3] K. Shimada, K. Kimura, S. Ichikawa, H. Ohta, and K. Aoki, "Phenomenon of labyrinth seal with low static pressure difference and large clearance," Visualization, vol. 6, no. 4, pp. 395405, 2003.

[4] Z. Lin, X. Wang, X. Yuan, N. Shibukawa, and T. Noguchi, "Investigation and improvement of the staggered labyrinth seal," Chinese Journal of Mechanical Engineering, vol. 28, no. 2, pp. 402-408, 2015.

[5] R. Kervistin and L. M. S. Seine, "Automatic control device of a labyrinth seal clearance in a turbo jet engine," France, 4668163, May 26, 1987. 
[6] U. Yucel and J. Y. Kazakia, "Analytical prediction techniques for axisymmetric flow in gas labyrinth seals," Journal of Engineering for Gas Turbines and Power, vol. 123, no. 1, pp. 255-257, 2001.

[7] L. D. Willey and B. Hills, "Labyrinth seal for a turbine bucket cover," US, 5890873, Apr. 6, 1999.

[8] H. Zhang, B. Xiong, X. An, C. Ke, and G. Wei, "Prediction on drag force and heat transfer of spheroids in supercritical water: A PR-DNS study," Powder Technology, vol. 342, pp. 99-107, 2019.

[9] H. Zhang, B. Xiong, X. An, C. Ke, and G. Wei, "Numerical investigation on the effect of the incident angle on momentum and heat transfer of spheroids in supercritical water," Computers \& Fluids, vol. 179, pp. 533-542, 2019.

[10] T. S. Kim and K. S. Cha, "Comparative analysis of the influence of labyrinth seal configuration on leakage behavior," Journal of Mechanical Science and Technology, vol. 23, no. 10, pp. 28302838, 2009.

[11] A. D. Vakili, A. J. Meganathan, M. Michaud, and S. Radhakrishnan, "An experimental and numerical study of labyrinth seal flow," ASME Paper GT2005-68224, 2005.

[12] B. Hodkinson, "Estimation of the Leakage through a Labyrinth Gland," Proceedings of the Institution of Mechanical Engineers, vol. 1-196, no. 1, pp. 283-288, 2016.

[13] T. Wu and C.-Y. Mi, "Numerical analysis on seal structure of high-speed gearbox lubrication system," Journal of the China Railway Society, vol. 36, no. 4, pp. 26-31, 2014.

[14] T. Hirano, Z. Guo, and R. G. Kirk, "Application of computational fluid dynamics analysis for rotating machinery - Part II: Labyrinth seal analysis," Journal of Engineering for Gas Turbines and Power, vol. 127, no. 4, pp. 820-826, 2005.

[15] R. Gao and G. Kirk, "CFD study on stepped and drum balance labyrinth seal," Tribology Transactions, vol. 56, no. 4, pp. 663671, 2013.

[16] G. Kirk and R. Gao, "Influence of preswirl on rotordynamic characteristics of labyrinth seals," Tribology Transactions, vol. 55, no. 3, pp. 357-364, 2012.

[17] Z. Li, J. Li, and Z. Feng, "Numerical investigations on the leakage and rotordynamic characteristics of pocket damper seals-Part II: Effects of partition wall type, partition wall number, and cavity depth," Journal of Engineering for Gas Turbines and Power, vol. 137, no. 3, pp. 032504-032513, 2015.

[18] Q. Deng, J. Niu, and Z. Feng, "Study on leakage flow characteristics of radial inflow turbines at rotor tip clearance," Science China Technological Sciences, vol. 51, no. 8, pp. 1125-1136, 2008.

[19] G. Y. Zhang, X. T. Yan, Y. Zhang, W. G. Zhao, and G. Z. Chen, "Study on the water-lubricated high-speed non-contact mechanical face seal supported by a disc spring," Brazilian Society of Mechanical Sciences Engineering, vol. 40, no. 7, p. 351, 2018. 


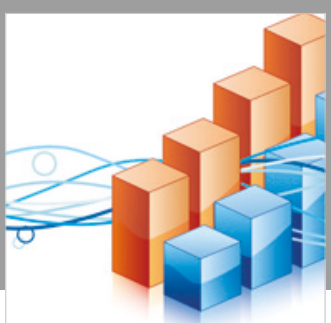

Advances in

Operations Research

\section{-n-m}
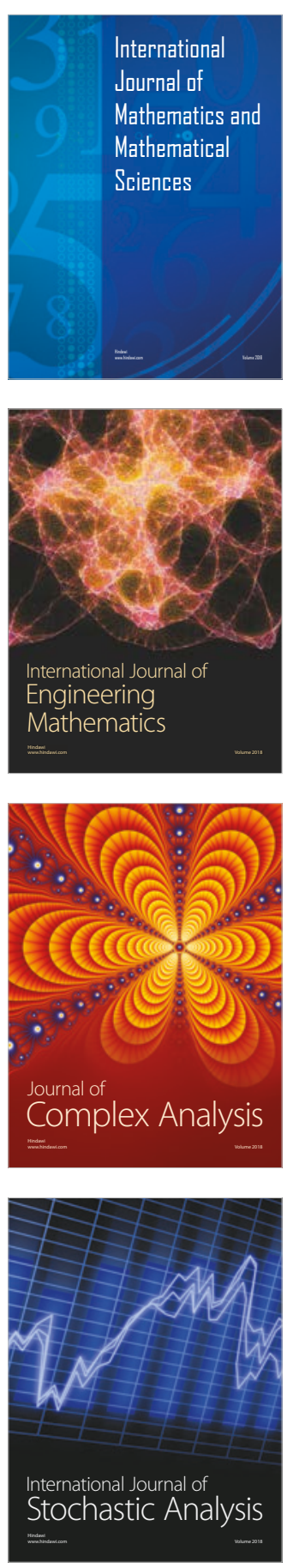
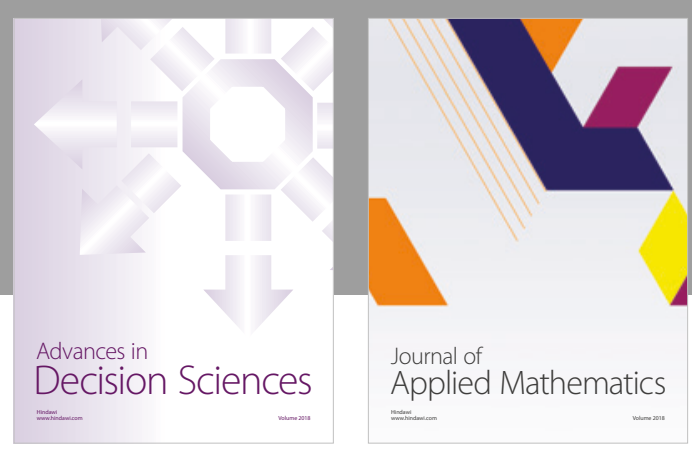

Journal of

Applied Mathematics
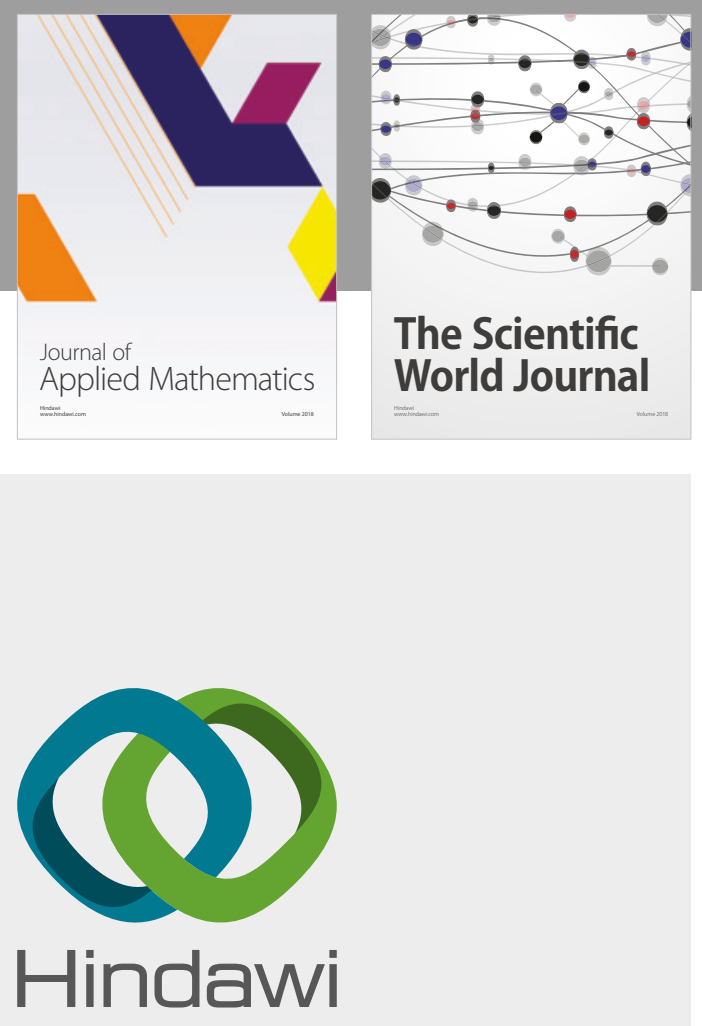

Submit your manuscripts at

www.hindawi.com

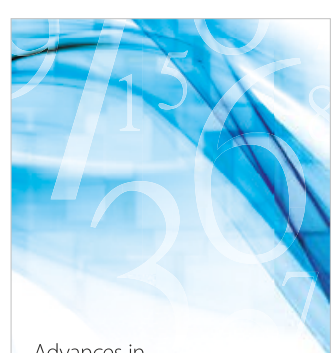

Advances in
Numerical Analysis
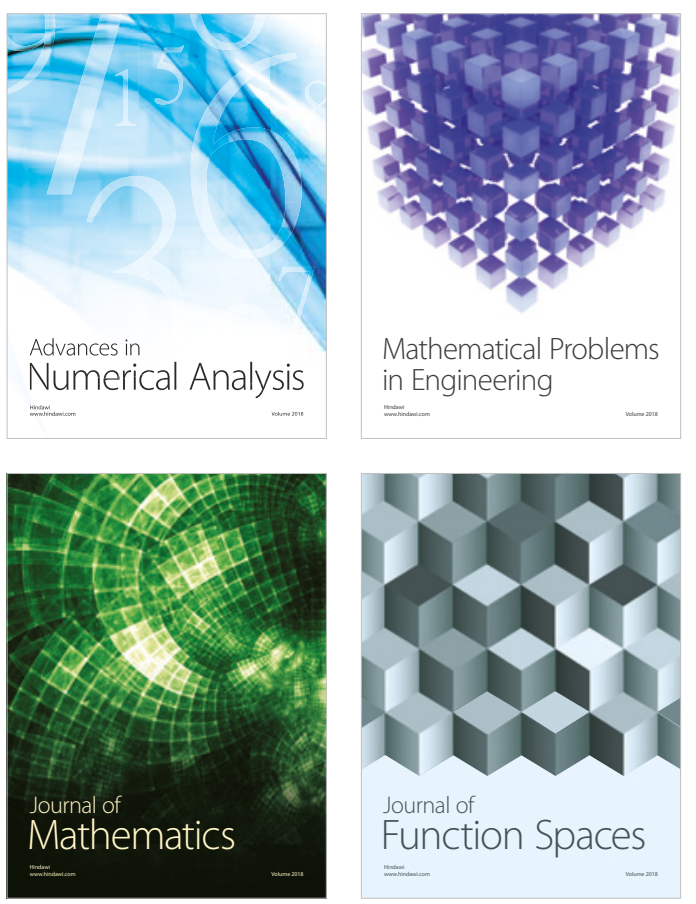

Mathematical Problems in Engineering

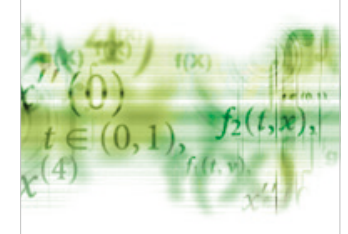

International Journal of

Differential Equations

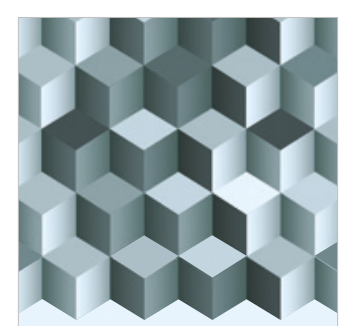

Journal of

Function Spaces
The Scientific

World Journal

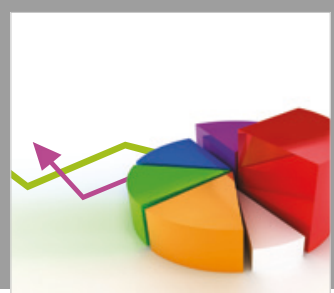

Journal of

Probability and Statistics
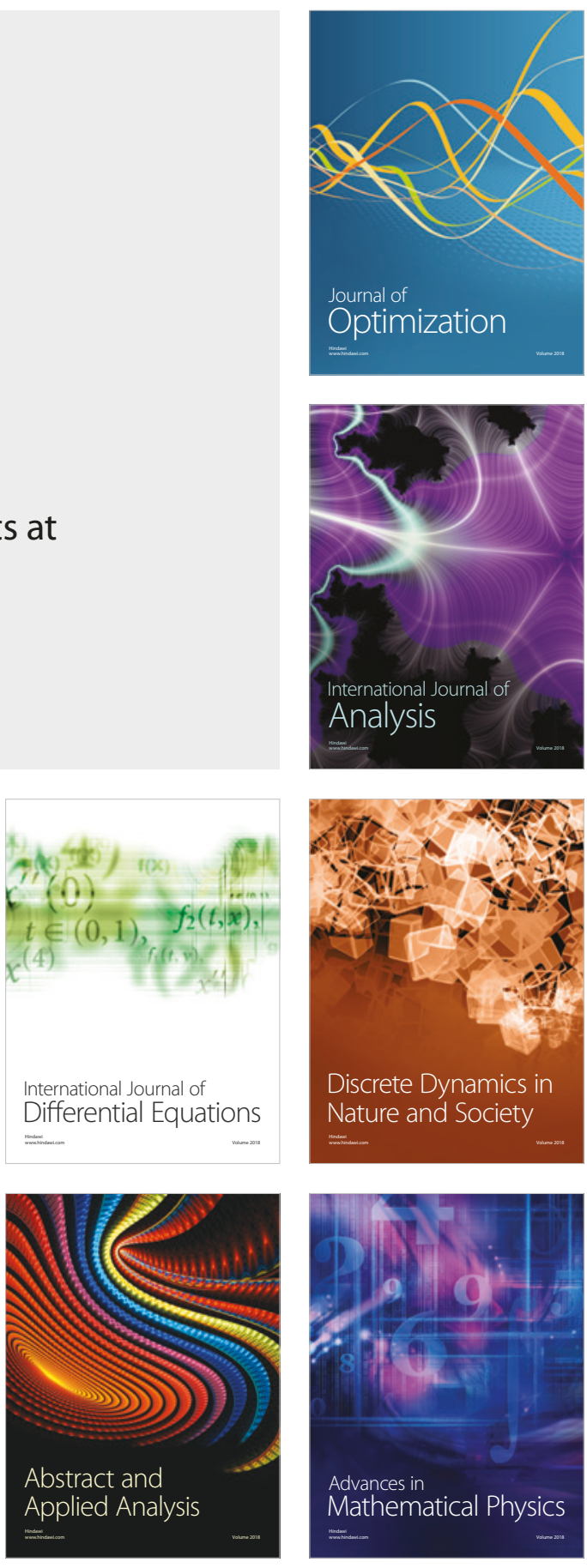